\title{
Boundary Values of Hyperfunction Solutions of Linear Partial Differential Equations
}

\author{
By \\ Hikosaburo Komatsu* and Takahiro KawAI
}

Let $P(x, D)$ be a linear partial differential operator with real analytic coefficients in a domain $V$ in $\mathbf{R}^{n+1}$ and let $S \subset V$ be a real analytic hypersurface non-characteristic with respect to $P(x, D)$. The purpose of this paper is to show that every hyperfunction solution $u$ of $P(x, D) u=0$ on one side of $V \backslash S$ has boundary values on $S$ which are hyperfunctions of $n$ variables on $S$.

This fact has been proved by H. Komatsu [6] and P. Schapira [8] in the case where $P(x, D)$ is elliptic. Their method applies with minor modifications to the general operators.

In $\S 1$ we show that the Cauchy-Kowalevsky theorem for the dual equation with the initial values on $S$ is equivalent to a theorem of division of hyperfunctions with supports in $S$ by the differential operator $P(x, D)$.

We define the boundary values in $\S 2$ and prove the uniqueness of hyperfunction solutions of the Cauchy problems.

\section{Division of Hyperfunctions with Supports in $\mathbf{S}$}

Let $P(x, D)$ be a linear differential operator of order $m$ with real analytic coefficients defined on a domain $V$ in $\mathbf{R}^{n+1}$ and let $S$ be an oriented real analytic hypersurface in $V$ non-characteristic with respect to $P(x, D)$.

We denote by $\mathscr{A}$ and $\mathscr{B}\left({ }^{\prime} \mathscr{A}\right.$ and ' $\left.\mathscr{B}\right)$ the sheaf of real analytic func-

Received December 15, 1970.

* Department of Mathematics, Faculty of Science, University of Tokyo, Hongo, Bunkyo-ku, Tokyo, Japan. Supported in part by the Matsunaga Science Foundation. 
tions and that of hyperfunctions on $V$ (on $S$ respectively). When $K$ is a compact set in $V$ (in $S$ ), the space $\mathscr{A}(K)(\mathscr{A}(K))$ has a natural (DFS)topology and its dual is identified with the space $\mathscr{B}_{K}(V)\left(\mathscr{B}_{K}(S)\right)$ of hyperfunctions with supports in $K$ under the inner product

$$
\begin{gathered}
\left\langle\varphi, f>=\int_{V} \varphi(x) f(x) d x, \varphi \in \mathscr{A}(K), f \in \mathscr{B}_{K}(V)\right. \\
\left(<\varphi, f>=\int_{S} \varphi\left(x^{\prime}\right) f\left(x^{\prime}\right) d \omega, \varphi \in^{\prime} \mathscr{A}(K), f \in^{\prime} \mathscr{B}_{K}(S)\right),
\end{gathered}
$$

where $d x(d \omega)$ denotes the Lebesgue measure on $V$ (on $S$ ).

Let $P^{\prime}(x, D)$ be the formal dual of $P(x, D)$. Then, $P(x, D)$ and $P^{\prime}(x, D)$ induce sheaf homomorphisms $P(x, D): \mathscr{B} \rightarrow \mathscr{B}$ and $P^{\prime}(x, D): \mathscr{A}$ $\rightarrow \mathscr{A}$ respectively. We denote by $\mathscr{B}^{P}$ and $\mathscr{A}^{P^{\prime}}$ the kernel sheaves, i.e., the sheaf of solutions of

$$
P(x, D) f=0, \quad f \in \mathscr{B},
$$

and that of solutions of

$$
P^{\prime}(x, D) \varphi=0, \quad \varphi \in \mathscr{A}
$$

respectively.

Theorem 1. Let $K$ be a compact set in $S$. Then, there is no nontrivial solution of (1) over $V$ with support in $K$ :

$$
\mathscr{B}_{K}^{P}(V)=0 .
$$

The quotient space $\mathscr{B}_{K}(V) / P \mathscr{B}_{K}(V)$ is identified with the dual of the (DFS)-space $\mathscr{A}^{P^{\prime}}(K)$.

Proof. Consider the complexes:

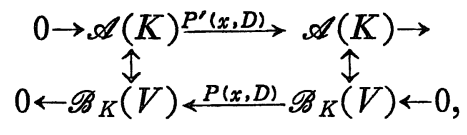

which are dual to each other in the sense that $\mathscr{A}(K)$ and $\mathscr{B}_{K}(V)$ with their natural $(D F S)$-and $(F S)$-topologies are the strong dual spaces of each other and that $P^{\prime}(x, D)$ and $P(x, D)$ are continuous linear operators dual 
to each other.

The 0 -th cohomology group of (4) is $\mathscr{A}^{P^{\prime}}(K)$ and the 1 -st cohomology group of (4) vanishes by the Cauchy-Kowalevsky theorem. In particular, $P^{\prime}(x, D)$ has a closed range. Thus it follows from Serre's lemma (see e.g. [5] Theorem 19) that $P(x, D)$ has a closed range and that the cohomology groups of (4) and (5) are the strong dual spaces of each other. Therefore, $\mathscr{B}_{K}(V) / P \mathscr{B}_{K}(V)$ is the dual of $\mathscr{A}^{P^{\prime}}(K)$ and $\operatorname{ker} P(x, D)=$ $\mathscr{B}_{K}^{P}(V)$ vanishes.

Let $C_{j}(x, D), j=1,2, \ldots, m$, be linear differential operators of order $m-j$ with real analytic coefficients on a neighborhood of $S$ for which $S$ is rcn-characteristic (e.g. $\left.C_{j}(x, D)=(\partial / \partial n)^{m-j}\right)$. Then the CauchyKcwa'evsky theorem yields the topological isomorphism

$$
\rho: \mathscr{A}^{P^{\prime}}(K) \approx^{\prime} \mathscr{A}(K)^{m}
$$

defined by

$$
\rho(\varphi)=\left(\left.C_{j}(x, D) \varphi\right|_{s}\right), \varphi \in \mathscr{A}^{P^{\prime}}(K) \text {. }
$$

we have, therefore, the dual isomorphism

$$
\rho^{\prime}: \mathscr{B}_{K}(S)^{m} \approx \mathscr{B}_{K}(V) / P \mathscr{B}_{K}(V) .
$$

Obviously $\rho$ can be extended by (7) to a continuous linear operator $\tilde{\rho}: \mathscr{A}(K) \rightarrow^{\prime} \mathscr{A}(K)^{m}$. Since the open mapping theorem holds for (DFS)spaces, the exact sequence

$$
0 \rightarrow^{\prime} \mathscr{A}(K)^{m} \stackrel{\rho^{-1}}{\longrightarrow} \mathscr{A}(K) \stackrel{P^{\prime}(x, D)}{\longrightarrow} \mathscr{A}(K) \rightarrow 0
$$

splits topologically and we have the topological isomorphism:

$$
\mathscr{A}(K) \approx^{\prime} \mathscr{A}(K)^{m} \oplus \mathscr{A}(K)
$$

defined by

$$
\varphi \mapsto\left(C_{j}(x, D) \varphi \mid s\right) \oplus P^{\prime}(x, D) \varphi .
$$

Correspondingly the dual exact sequence

$$
0 \leftarrow^{\prime} \mathscr{B}_{K}(S)^{m} \stackrel{\left(\rho^{-1}\right)^{\prime}}{\mathscr{B}_{K}}(V) \stackrel{P(x, D)}{\longleftarrow} \mathscr{B}_{K}(V) \leftarrow 0
$$

splits topologically. 
Since $\tilde{\rho}$ is the composite of the differential operators $\left(C_{j}(x, D)\right)$ and the restriction to $S$, the dual $\tilde{\rho}^{\prime}: \mathscr{B}_{K}(S)^{m} \rightarrow \mathscr{B}_{K}(V)$ is the mapping $\left(f_{j}\right) \rightarrow$ $\sum_{j=1}^{m} C_{j}^{\prime}(x, D)\left(f_{j} \otimes \delta_{S}\right)$, where $C_{j}^{\prime}(x, D)$ is the formal dual of $C_{j}(x, D)$ and $f_{j} \otimes \delta_{s}$ is the hyperfunction on $V$ defined by

$$
\left\langle f_{j} \otimes \delta_{S}, \varphi>=\int_{S} f_{j}\left(x^{\prime}\right) \varphi\left(x^{\prime}\right) d \omega, \varphi \in \mathscr{A}(K) .\right.
$$

Consequently, each $f \in \mathscr{B}_{K}(V)$ is uniquely decomposed as

$$
f=\sum_{j=1}^{m} C_{j}^{\prime}(x, D)\left(f_{j} \otimes \delta_{S}\right)+P(x, D) g,
$$

where $f_{j} \in \mathscr{B}_{K}(S)$ and $g \in \mathscr{B}_{K}(V)$. Under this correspondence we have a topological isomorphism

$$
\mathscr{B}_{K}(V) \approx^{\prime} \mathscr{B}_{K}(S)^{m} \oplus \mathscr{B}_{K}(V) .
$$

In particular, the inverse $\left(\rho^{\prime}\right)^{-1}: \mathscr{B}_{K}(V) / P \mathscr{B}_{K}(V) \approx^{\prime} \mathscr{B}_{K}(S)^{m}$ of isomorphism (8) is the mapping which takes the class of $f$ to $\left(f_{j}\right)$ in the decomposition (14). Obviously $f_{j}$ depend on the choice of $C_{j}(x, D)$. However, the sum $\sum C_{j}^{\prime}(x, D)\left(f_{j} \otimes \delta_{s}\right)$ and $P(x, D) g$ do not depend on $C_{j}(x, D)$ because neither $\operatorname{im} \rho^{-1}=\mathscr{A}^{P^{\prime}}(K)$ nor ker $\tilde{\rho}$ depends on $C_{j}(x, D)$.

The uniqueness of the decomposition shows that the components $f_{j}$ and $g$ are independent of the compact set $K$ which contains the support of $f$. Namely we have an isomorphism

$$
\Gamma_{*}\left(\left.\mathscr{H}_{S}^{0}(\mathscr{B})\right|_{s}, S\right) \approx \Gamma_{*}\left({ }^{\prime} \mathscr{B}, S\right)^{m} \oplus \Gamma_{*}\left(\left.\mathscr{H}_{S}^{0}(\mathscr{B})\right|_{s}, S\right)
$$

which preserves the support, where $\Gamma_{*}$ denotes the space of sections with compact supports and $\left.\mathscr{H}_{S}^{0}(\mathscr{B})\right|_{S}$ the restriction to $S$ of the sheaf of sections of $\mathscr{B}$ with supports in $S$.

Let us denote $\left.\mathscr{H}_{S}^{0}(\mathscr{B})\right|_{S}$ by $\mathscr{B}_{S}$ for short. Since $\mathscr{B}_{S}$ and ' $\mathscr{B}$ are flabby, it follows that the isomorphism is extended to a sheaf isomorphism (see e.g. [4] Lemma 2.3). Thus we have proved the following theorem.

Theorem 2. If $C_{j}^{\prime}(x, D), j=1, \ldots, m$, are linear differential operators of order $m-j$ with real analytic coefficients on a neighborhood of $S$ for which $S$ is non-characteristic, then we have a sheaf isomorphism 


$$
\mathscr{B}_{S} \approx^{\prime} \mathscr{B}^{m} \oplus \mathscr{B}_{S}
$$

defined by

$$
f=\sum_{j=1}^{m} C_{j}^{\prime}(x, D)\left(f_{j} \otimes \delta_{S}\right)+P(x, D) g,
$$

where $f \in \mathscr{B}_{S}, f_{j} \in \mathscr{B}^{\prime}$ and $g \in \mathscr{B}_{S}$. The last component $g$ does not depend on the choice of $C_{j}^{\prime}(x, D)$.

In particular, there is no non-trivial solution $g \in \mathscr{B}^{P}(V)$ with support in $S$ :

$$
\mathscr{B}_{S}^{P}(V)=0 .
$$

This theorem means that on division by $P(x, D)$ each $f \in \mathscr{B}_{S}$ has a unique quotient $g \in \mathscr{B}_{S}$ and a remainder $\sum C_{j}^{\prime}(x, D)\left(f_{j} \otimes \delta_{S}\right)$ with $f_{j} \in \mathscr{B}$. We have derived this from the Cauchy-Kowalevsky theorem via the duality of $\mathscr{A}(K)$ and $\mathscr{B}_{K}(V)$ and that of $\mathscr{A}(K)$ and ${ }^{\prime} \mathscr{B} K(S)$. Conversely Theorem 2 implies the exactness of (12) and hence that of (9). Thus Theorem 2 of division is equivalent to the Cauchy-Kowalevsky theorem.

\section{Boundary Values of Hyperfunction Solutions}

Let $W$ be an open subset of $V$. We have the following commutative diagram:

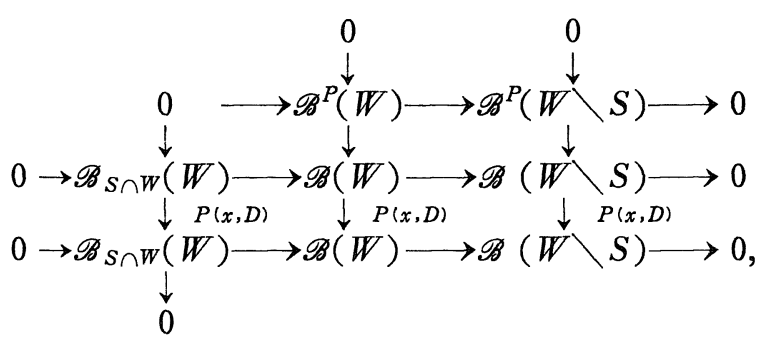

where $\mathscr{B}_{S \cap W}(W)$ denotes the space of hyperfunctions on $W$ with supports in $S \cap W$. Since $\mathscr{B}$ is flabby, the last two rows are exact; the last two columns are exact by the definition; the 0 -th cohomology group of the first row and that of the first column vanish since there is no non-trivial solution with support in $S \cap W$.

For the remaining cohomology groups we have a natural homomor- 
phism

$$
b: \mathscr{B}^{P}(W \backslash S) / \mathscr{B}^{P}(W) \rightarrow \mathscr{B}_{S \cap W}(W) / P \mathscr{B}_{S \cap W}(W) .
$$

Let $u \in \mathscr{B}^{P}(W \backslash S)$ and let $\bar{u}$ be an extension in $\mathscr{B}(W)$. Since $P(x, D) \tilde{u}$ $=0$ on $W \backslash S, P \tilde{u}$ belongs to $\mathscr{B}_{S \cap W}(W)$. If $\tilde{u}_{1}$ is another extension of $u, \tilde{u}-\tilde{u}_{1}$ belongs to $\mathscr{B}_{S \cap W}(W)$. Therefore the class of $P \tilde{u}$ in $\mathscr{B}_{S \cap W}(W) / P \mathscr{B}_{S \cap W}(W)$ is determined uniquely by $u$. If $u$ is the restriction to $W \backslash S$ of a $\tilde{u} \in \mathscr{B}^{P}(W)$, we have $P \tilde{u}=0$. Thus we can define a homomorphism $b$ which assigns for the class of $u \in \mathscr{B}^{P}(W \backslash S)$ the class of $P \tilde{u} \in \mathscr{B}_{S \cap W}(W)$.

\section{Theorem 3. The homomorphism}

$$
b: \quad \mathscr{B}^{P}(W \backslash S) / \mathscr{B}^{P}(W) \rightarrow \mathscr{B}_{S \cap W}(W) / P \mathscr{B}_{S \cap W}(W)
$$

is injective for any open set $W$ in $V$ and commutes with restrictions. $b$ is surjective if and only if

$$
P(x, D) \mathscr{B}(W) \supset \mathscr{B}_{S \cap W}(W) .
$$

Proof. By the definition it is clear that $b$ commutes with restrictions. To prove the injectivity, let $P \tilde{u}=P u_{1}$ for a $u_{1} \in \mathscr{B}_{S \cap W}(W)$. Since $\tilde{u}-u_{1} \in \mathscr{B}^{P}(W)$ and its restriction to $W \backslash S$ is equal to $u$, the class of $u$ is zero.

Let $b$ be surjective. Then, for each $g \in \mathscr{B}_{S \cap W}(W)$ there exist $h \in$ $\mathscr{B}_{S \cap W}(W)$ and $\tilde{u} \in \mathscr{B}(W)$ such that $g+P h=P \tilde{u}$. Thus $\mathscr{B}_{S \cap W}(W) \subset P \mathscr{B}(W)$.

Conversely suppose that for each $g \in \mathscr{B} S \cap W(W)$ there is a $\tilde{u} \in \mathscr{B}(W)$ such that $g=P \tilde{u}$. Then, the restriction $u$ of $\tilde{u}$ belongs to $\mathscr{B}^{P}(\mathbb{W} \backslash S)$. Therefore $b$ is surjective.

It is known that (22) holds if the coefficients of $P(x, D)$ are constants or if $P(x, D)$ is elliptic.

Now, let $\omega$ be an open set of $S$ and let $W \supset W^{\prime}$ be two open sets in $V$ with $S \cap W=S \cap W^{\prime}=\omega$. The restriction $\mathscr{B}^{P}(W \backslash S) \rightarrow \mathscr{B}^{P}\left(W^{\prime} \backslash S\right)$ induces a homomorphism

$$
r: \quad \mathscr{B}^{P}(W \backslash S) / \mathscr{B}^{P}(W) \rightarrow \mathscr{B}^{P}\left(W^{\prime} \backslash S\right) / \mathscr{B}^{P}\left(W^{\prime}\right) .
$$


Since $\mathscr{B}_{S \cap W}(W) / P_{\mathscr{B}_{S \cap W}}(W)=\mathscr{B}_{S \cap W^{\prime}}\left(W^{\prime}\right) / P \mathscr{B}_{S \cap W^{\prime}}\left(W^{\prime}\right)$ and since the injections $b_{W}$ and $b_{W}$, commute with $r$, it follows that $r$ is injective.

$r$ is surjective if and only if $\mathscr{B}^{P}\left(W^{\prime} \backslash S\right)=\mathscr{B}^{P}(W \backslash S) \mid W^{\prime} \backslash S$ $+\left.\mathscr{B}^{P}\left(W^{\prime}\right)\right|_{W 八 s}$ and this holds if

$$
H^{1}\left(W, \mathscr{B}^{P}\right)=0
$$

by the Mayer-Vietoris theorem.

It is also known that (24) holds for any open set $W$ if the coefficients of $P(x, D)$ are constants or if $P(x, D)$ is elliptic.

Taking the inductive limit with respect to the open neighborhoods of $\omega$, we have the injection

$$
b: \quad\left(\mathscr{B}_{+}^{P}(\omega) \oplus \mathscr{B}_{-}^{P}(\omega)\right) / \mathscr{B}^{P}(\omega) \rightarrow \mathscr{B}_{S}(\omega) / P \mathscr{B}_{S}(\omega),
$$

where $\mathscr{B}_{+}^{P}(\omega)\left(\mathscr{B}_{-}^{P}(\omega)\right)$ denotes the space of germs of solutions on $W \backslash S$ which vanish on the negative (positive) side of $S . \mathscr{B}_{ \pm}^{P}$ are sheaves over $S$ which describe the boundary behavior of solutions outside $S$.

It follows from Theorem 3 that $b$ in (25) is surjective if and only if

$$
P(x, D) \mathscr{B}(\omega) \supset \mathscr{B}_{S}(\omega) .
$$

Furthermore, noticing that the sheaf associated with the presheaf $\left(\mathscr{B}_{+}^{P}(\omega) \oplus \mathscr{B}_{-}^{P}(\omega)\right) / \mathscr{B}^{P}(\omega)$ is the restriction $\left.\mathscr{H}_{S}^{1}\left(\mathscr{B}^{P}\right)\right|_{s}$ to $S$ of the first derived sheaf with support in $S$ (see $[4]$ ), we have the injection

$$
b:\left.\quad \mathscr{H}_{S}^{1}\left(\mathscr{B}^{P}\right)\right|_{S} \rightarrow \mathscr{B}_{S} / P_{\mathscr{B}}{ }_{S}
$$

which is surjective if and only if

$$
\left.P(x, D) \mathscr{B}\right|_{S} \supset \mathscr{B}_{S} .
$$

Obviously (28) is satisfied if $P(x, D)$ is locally solvable on $S$, i.e. if

$$
P(x, D): \mathscr{B}(x) \rightarrow \mathscr{B}(x) \text { is surjective for } x \in S .
$$

This is known for operators with constant coefficients or of elliptic type. Moreover, T. Kawai [3] proves the existence of local elementary solutions and hence the local solvability of operators $P(x, D)$ of simple characteristics with real principal parts. Thus (27) is an isomorphism for such operators. Combining this with the isomorphism $\mathscr{B}_{S} / P \mathscr{B}_{S} \approx^{\prime} \mathscr{B}^{m}$ 
given in Theorem 2, we have an isomorphism

$$
\left.\mathscr{H}_{S}^{1}\left(\mathscr{B}^{P}\right)\right|_{S} \approx^{\prime} \mathscr{B}^{m} .
$$

Definition. Let $W$ be an open set in $V$, let $\omega=S \cap W$ and let $W+$ be the positive part of $W \backslash S$. For each solution $u \in \mathscr{B}^{P}\left(W_{+}\right)$we define its boundary values $\left(f_{j}\right) \in^{\prime} \mathscr{B}(\omega)^{m}$ on $S$ to be the image of $u$ under the composite of mappings $\mathscr{B}^{P}\left(W_{+}\right) \rightarrow \mathscr{B}_{+}^{P}(\omega) \rightarrow\left(\mathscr{B}_{+}^{P}(\omega) \oplus \mathscr{B}_{-}^{P}(\omega)\right) / \mathscr{B}^{P}(\omega) \stackrel{b}{\longrightarrow}$ $\mathscr{B}_{S}(\omega) / P \mathscr{B}_{S}(\omega) \rightarrow^{\prime} \mathscr{B}(\omega)^{m}$, where the last mapping is the isomorphism obtained in Theorem 2 as an extension of $\left(\rho^{\prime}\right)^{-1}$ in (8). In other words, $\left(f_{j}\right) \in^{\prime} \mathscr{B}(\omega)^{m}$ is the unique $m$-tuple of hyperfunctions on $\omega$ which satisfy

$$
P(x, D) \bar{u}=\sum_{j=1}^{m} C_{j}^{\prime}(x, D)\left(f_{j} \otimes \delta_{S}\right)
$$

for an extension $\tilde{u} \in \mathscr{B}(W)$ vanishing on the negative side of $W \backslash S$.

As we remarked earlier, the extension $\tilde{u}$ which satisfies (31) is uniquely determined by $u$ and does not depend on the choice of $C_{j}(x, D)$, so that we call $\tilde{u}$ the canonical extension of $u$.

Let $\theta_{S}$ be the characteristic function of $W_{+}$in $W$. Then, there are unique linear differential operators $B_{j}(x, D), j=1, \ldots, m$, of order $j-1$ with real analytic coefficients in a neighborhood of $S$ such that $S$ is noncharacteristic and that

$$
\begin{gathered}
P(x, D)\left(\theta_{S}(x) u(x)\right)-\theta_{S}(x)(P(x, D) u(x)) \\
=\sum_{j=1}^{m} C_{j}^{\prime}(x, D)\left(B_{j}(x, D) u(x)\left(1 \otimes \delta_{S}\right)\right) \\
=\sum_{j=1}^{m} C_{j}^{\prime}(x, D)\left(\left.\left(B_{j}(x, D) u(x)\right)\right|_{s} \otimes \delta_{S}\right)
\end{gathered}
$$

for any $u \in \mathscr{A}(W)$ or more generally for any $u \in \mathscr{B}(W)$ which is real analytic in the normal direction on $S$ (see $[1]$ for the real analyticity in parameter and the restrictions of hyperfunctions to submanifolds).

Conversely if $B_{j}(x, D), j=1, \ldots, m$, are linear differential operators of order $j-1$ with real analytic coefficients for which $S$ is non-characteristic, we can find linear differential operators $C_{j}(x, D)$ of order $m-j$ such that $S$ is non-characteristic and that (32) holds. This is only a local formulation of Green's formula. 
Therefore, if $u$ is the restriction to $W_{+}$of a solution $u_{1} \in \mathscr{A}^{P}(W)$ we have

$$
f_{j}=\left.B_{j}(x, D) u_{1}\right|_{s}, j=1, \ldots, m .
$$

This holds also for the restriction $u$ of a solution $u_{1} \in \mathscr{B}^{P}(W)$, because $u_{1}$ is real analytic in the normal direction on $S$ by Sato's fundamental theorem of analyticity (see [1]).

Taking this into account we will write the boundary values

$$
f_{j}=\left.B_{j}(x, D) u\right|_{s_{+}}, \quad j=1, \ldots, m .
$$

Similarly we can define the boundary values $\left.B_{j}(x, D) u\right|_{s_{-}}$of solutions $u$ on the negative side of $W \backslash S$. The following is clear from the definition.

Theorem 4. A solution $u \in \mathscr{B}^{P}(W \backslash S)$ is extended to a solution $u \in \mathscr{B}^{P}(W)$ if and only if

$$
\left.B_{j}(x, D) u\right|_{s_{+}}=\left.B_{j}(x, D) u\right|_{s_{-}}, j=1, \ldots, m .
$$

This may be regarded as a generalization of the classical Painlevé theorem.

If the operator $P(x, D)$ is locally solvable on $S$ or if (28) holds, then the isomorphism (30) shows that the Plemelj problem

$$
\left.B_{j}(x, D) u\right|_{s_{+}}-\left.B_{j}(x, D) u\right|_{s_{-}}=f_{j}, j=1, \ldots, m
$$

has a local solution $u \in \mathscr{B}_{+}^{P}(x) \oplus \mathscr{B}_{-}^{P}(x)$ for any $f_{j} \in \mathscr{B}(x)$ on $S$.

Lastly the Holmgren theorem by T. Kawai [2] and P. Schapira [7] asserts that

$$
\mathscr{B}_{+}^{P}(\omega) \cap \mathscr{B}^{P}(\omega)=\{0\} \text { and } \mathscr{B}_{-}^{P}(\omega) \cap \mathscr{B}^{P}(\omega)=\{0\} .
$$

Therefore the mapping $\mathscr{B}_{+}^{P}(\omega) \rightarrow^{\prime} \mathscr{B}(\omega)^{m}$ is injective. Thus we have

Theorem 5. A solution $u \in \mathscr{B}^{P}\left(W_{+}\right)$on the positive side of $W \backslash S$ vanishes in a neighborhood of $\omega=W \cap S$ if and only if the boundary values $\left.B_{j}(x, D) u\right|_{s}$ vanish for all $j=1, \ldots, m$. 


\section{References}

[1] Kashiwara, M., On the structure of hyperfunctions (following M. Sato), Sûgaku no Ayumi, 15 (1970), 9-72 (Japanese).

[2] Kawai, T., The theory of Fourier transforms in the theory of hyperfunctions and its applications, Master's thesis, Univ. of Tokyo, 1970, partly reprinted in Surikaiseki Kenkŷ̂sho Kôkyûroku, No. 108 Kyoto Univ. pp. 84-288 (Japanese).

[3] _ - Construction of a local elementary solution for linear partial differential operators (I), Proc. Japan Acad. 47 (1971), 19-23.

[4] Komatsu, H., Relative cohomology of sheaves of solutions of differential equations, Síminaire Lions-Schwartz, 1966-67, reprinted in Symposium on Algebraic Geometry Expecting Applications to Hyperfunctions, Katata, 1969.

[5] _ _ _ Projective and injective limits of weakly compact sequences of locally convex spaces, J. Math. Soc. Japan, 19 (1967), 366-383.

[6] - Boundary values for solutions of elliptic equations, Proc. Intern. Conf. Functional Analysis and Related Topics, 1969, Univ. Tokyo Press, 1970, pp. 107121.

[ 7 ] Schapira, P., Théorème d'unicité de Holmgren et opérateurs hyperboliques dans l'espace de hyperfonctions, to appear in Anais Acad. Brasil. Sci.

[8] - Hyperfonctions et problèmes aux limites elliptiques, to appear in Ann. Inst. Fourier. 